\title{
QOS ISSUES IN COMPLEX-BASED HIGH ALTITUDE PLATFORM COMMUNICATION SYSTEMS
}

The use of High Altitude Platforms is a promising solution towards the integration of different wireless networks. Complex-based High Altitude Platform communication systems can offer high quality wireless services combined with enhanced bandwidth efficiency. Inter-system handover and power control are major factors affecting the provided quality of service. The impact of these factors under strong fading environments is studied in the present work.

\section{Introduction}

High Altitude Platforms (HAPs) are unmanned airships that carry communicational equipment and provide a great variety of wireless services in a certain area on the ground. Local Multipoint Distribution Services (LMDS) including Broadband Fixed Wireless Access (BFWA), navigation, localization, 3G and beyond, environmental surveillance are some of the potentials this technology can provide [2]. The advantages of the use of HAPs are more evident in cases of areas suffering from deficient telecommunication ground infrastructures or in cases of emerging need for wireless coverage, exceeding the capacity of fixed networks. Systems of multiple HAPs are of great interest, as they enhance network performance, offering flexible architectures. They can be deployed as part of extended heterogeneous networks and they can contribute to the integration of different wireless networks. Two main issues are addressed in the present work: the effects of inter-HAPs handover and fairness in downlink broadcasting quality for different groups of subscribers.

\section{System Description}

In the proposed work, a constellation of four HAPs in the stratosphere serves an area on the ground. The platforms share the same bandwidth and they illuminate the area using omni-directional antennas. Users on the ground may be either fixed or mobile. In order for the system to be feasible, users have to carry directional antennas. The directionality makes possible the usage of common resources by the HAPs transmitting systems, as analyzed in [1]. There, by means of interference analysis, it is proved that sufficient levels of Carrier to Interference plus Noise Ratio (CINR) are achieved. Each user is served by a single platform. This is called the main HAP. The other platforms of the constellation produce interference (interfering $H A P S$ ). As a result

$$
C I N R=\frac{P_{C \_ \text {received }}^{\text {main }}}{N_{F}+\sum_{k \in I} P_{I_{-} \text {received, } k}}
$$

where the nominator is the received signal power from the main HAP, $N_{F}$ is the noise floor and $P_{I-\text { received, } k}$ is the received power from the $k$-th interfering HAP. The interfering signal is received via the side lobes of the user antenna. Figure 1 (a) shows the system's topology.

Although users can be served by any HAP with satisfactory levels of quality of service, only one HAP at a time can achieve optimal performance. Consequently, enhanced performance could be achieved if it was possible for the users to choose the HAPs that offer optimal coverage conditions. This could be realized by means of constant monitoring. The received power of the pilot channels of the HAPs could be the monitored parameters. One major factor that enables this operation is that because of the nature of the architecture, the users can be easily redirected from one HAP to another. It is important that there is a mechanism to control inter-HAP handover properly. The system must be protected from unnecessary handovers. For example, there must be a minimum

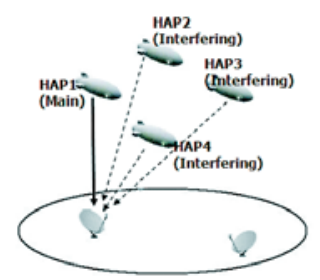

(a)

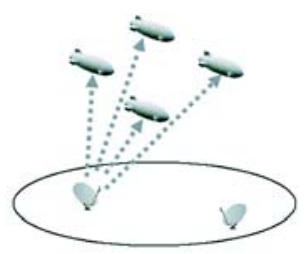

(b)
Fig.1 (a)Multiple HAPs coexistence (interference scenario), (b)Inter-HAP handover

\footnotetext{
* Konstantinos Birkos, Theofilos Chrysikos, Stavros Kotsopoulos

Wireless Telecommunication Laboratory, Department of Electrical \& Computer Engineering, University of Patras, Greece,

E-mail: kmpirkos@ece.upatras.gr
} 
number of samples in which a HAP is detected as the optimal one before it is selected as the main HAP, in order to avoid ping-pong effects.

In case of voice and video-oriented networks, the downlink broadcast performance is another matter of interest. In particular, for systems of multiple HAPs, a power control mechanism would guarantee fairness in terms of certain performance evaluation criteria for groups of users served by different platforms. Inter-HAP handover may guarantee enhanced uniformity of coverage and outage probability for the area of coverage but it cannot always offer optimal performance without proper power control. This is evident in packet-transmission-based systems in which delays, throughput and consumed energy depend on the number of receiving nodes. These issues are addressed in the present work.

A send-and-wait Automatic-Repeat-ReQuest (ARQ) scheme is considered in the downlink for the broadcasting feature. It means that each transmitting platform repeatedly retransmits each packet until all nodes served by it receive it correctly. The average number of retransmissions per packet is a function of the probability of successful reception and the number of receiving nodes. With the inter-HAP handover enabled, different platforms serve different numbers of subscribers according to optimal coverage requirements. Therefore, when uniform power allocation among HAPs is implied, differences in average end-to-end delays and energy-per-successfulpacket-reception will be observed. As shown next, a scheme able to re-allocate power among HAPs transmitters could yield in the equalization of delays for users served by different platforms.

Two factors affect the probability of successful packet reception: the ratio of bit energy to power spectral density $\mathrm{Eb} / \mathrm{No}$ and the packet length. In the examined system, $\mathrm{Eb} / \mathrm{No}$ is a function of the user's position, the modulation, the bit rate, the transmitting power of each platform and which platform the user is served by The probability of successful reception ps is given by

$$
p_{s}=(1-B E P)^{L}
$$

where $B E P$ is the bit error probability and $L$ is the packet length in bits. The packet reception is a Bernoulli procedure as each packet is correctly received with probability $p_{s}$ or incorrectly received with packet error probability $P E P=1-p s$. As a result, the geometric distribution describes the number of trials in order to achieve a successful reception. Following the analysis presented in [15], the average number of transmissions per packet is expressed as

$$
N_{\text {tr_ave }}=\sum_{n=1}^{N_{G}}\left(\begin{array}{c}
N_{C} \\
n
\end{array}\right) \cdot \frac{(-1)^{n+1}}{1-P E P^{n}}
$$

where $N_{C}$ is the number of receiving nodes. The average number of transmissions affects the average service time according to the following formula

$$
t_{\text {serv_ave }}=N_{\text {tr_ave }} \cdot\left(t^{\text {trans }}+R T T\right)
$$

where $t_{\text {trans }}$ is the transmission delay and $R T T$ is the round trip time.

\section{Channel modelling}

The received signal is given by $y(t)=h(t) s(t-\tau)+n(t)$, where $h(t)$ is the complex channel function that inserts the multiplicative attenuation effects on the transmitted signal $s(t-\tau)$, which suffers from an average propagation delay $\tau$, and $n(t)$ is the additive noise effect. The propagation delays in each path (therefore for each component of the signal) are considered relatively small compared to the symbol period of the modulation method. Therefore the fading is considered flat or frequency non-selective.

A worst-case channel scenario is considered, assuming heavy obstruction and mostly in-building user movement, so that a strong LOS component is missing. In this case, Rayleigh fading is most appropriate for the description of the fading effects due to multipath propagation (small-scale fading). The received signal consists of a sum of reflected components (NLOS case) and the instantaneous amplitude of the received signal follows a Rayleigh distribution, whereas the instantaneous received power follows an exponential distribution. For the shadowing effects (large-scale fading) the lognormal distribution is considered as most appropriate and is more commonly used [3]. A mixed Rayleigh-lognormal distribution is therefore used to represent both shadowing and fast fading effects $[4,5]$. The Rayleigh-lognormal model, also known as Suzuki model, has been evaluated by HAPs surveys as one of the most appropriate for the study of HAPs systems, especially in 'bad channel performance' scenarios [6]. It has also been proposed as a model for the description of the satellite channel [7].

The joint pdf of the Rayleigh-lognormal fading is an integrated product of the conditional joint Rayleigh-lognormal pdf and the lognormal pdf. The following equations are expressed in terms of instantaneous received power [8]:

$$
p(s)=\int_{0}^{\infty} f_{R \mid \Omega}(s) f_{\Omega}(\Omega) d \Omega
$$

Where $f_{R \mid \Omega}$ is the conditional joint Rayleigh-lognormal pdf. The exponential distribution originating from the Rayleigh 'component' of the 'composite' Rayleigh-Lognormal (RL) fading model expresses the instantaneous received power $\mathrm{s}$ as it varies around a mean value $\Omega$ which is calculated by path loss models. In a case of multipath-only fading ('pure' Rayleigh fading), this would be the average received power. In this study, however, the shadow fading is also taken into consideration, therefore $\Omega$ represents the local mean value, which in long term also varies in the accordance of the lognormal distribution:

$$
\begin{aligned}
& f_{R \mid \Omega}(s)=\frac{1}{\Omega} e^{\left(-\frac{s}{\Omega}\right)} \\
& f_{\Omega}(\Omega)=\frac{1}{\sqrt{2 \pi \sigma_{s h} \Omega}} e^{\left\{-\frac{1}{2}\left(\frac{\ln (\Omega)-\mu_{s h}}{\sigma_{s h}}\right)^{2}\right\}}
\end{aligned}
$$

$f_{\Omega}(\Omega)$ : lognormal distribution which expresses the long-term variation of the (local) mean value $\Omega$. In order to express the standard shadow deviation in $\mathrm{dB}$, the following adjustment needs to be 
made: $\sigma_{d B}=\frac{10}{\ln (10)} \sigma_{s h}$, therefore: $\sigma_{s h}=\frac{\ln (10)}{10} \sigma_{d B}$. For the mean value, which stands for the area mean power, it is suggested in [9] that it is dependent on the distance between the transmitter and the receiver: $\mu_{s h}=\mu(r)-\beta \ln \frac{r}{R}, \beta$ being the path loss exponent, $r$ the distance between transmitter and receiver, $R$ the cell radius and $\mu_{R}$ the mean value (area mean power) at the edge of the cell, where $r=R$. It has been estimated that in a dynamic range cell for Rayleigh small-scale fading, the area mean power at the edge of the cell is around $60-80 \mathrm{dBm}$, it is safe therefore to presume a value of $70 \mathrm{dBm}$ for $\mu_{R}$. The pdf of the composite Rayleigh-lognormal fading can be expressed - in terms of instantaneous received power - as:

$$
p(s)=\int_{0}^{\infty} \frac{1}{\Omega} e^{\left(-\frac{s}{\Omega}\right)} \frac{1}{\sqrt{2 \pi \varphi \sigma_{d B} \Omega}} e^{\left\{-\frac{1}{2}\left(\frac{\ln (\Omega)-\mu(r)}{\varphi \sigma_{d B}}\right)^{2}\right\}} d \Omega
$$

where $\varphi=\frac{\ln (10)}{10}$.

Since the instantaneous received power follows a certain pdf, it can be assumed that the intantaneous received SNR follows the same pdf, based on the fact that it is a 'scaled' depiction of the received power [10]:

$$
\mathrm{p}(\gamma)=\int_{0}^{\infty} \frac{1}{\Gamma} e^{\left(-\frac{\gamma}{\Gamma}\right)} \frac{1}{\sqrt{2 \pi \varphi \sigma_{d B} \Gamma}} e^{\left\{-\frac{1}{2}\left(\frac{\ln (\Gamma)-\mu(r)}{\varphi \sigma_{d B}}\right)^{2}\right\}} d \Gamma
$$

where $\gamma$ expresses the instantaneous SNR and $\Gamma$ the average SNR.

The Outage Probability is the probability that the received SNR falls below a certain threshold $\gamma_{t h}$. It is derived out of the CDF (Rayleigh):

$$
\begin{aligned}
& F(\gamma)=1-e^{\left(-\frac{\gamma}{\Gamma}\right)} \\
& P_{\text {out }}(r \mid \Gamma)=\operatorname{Pr}\left[\gamma<\gamma_{t h}\right]=F\left(\gamma_{t h}\right)=1-e^{\left(-\frac{\gamma_{t h}}{\Gamma}\right)}
\end{aligned}
$$

This is the conditional outage probability since we have only taken into consideration the Rayleigh component of the composite fading model. It has been calculated for a given $\Gamma$ and it is also dependent on the distance between transmitter-receiver [9]. Taking into account the lognormal variation of $\Gamma$, the Outage Probability is dependent only on the distance $r$ and is expressed as:

$$
P_{\text {out }}(r)=\int_{0}^{\infty} 1-e^{\left(-\frac{\gamma_{\text {th }}}{\Gamma}\right)} \frac{1}{\sqrt{2 \pi \varphi \sigma_{d B} \Gamma}} e^{\left\{-\frac{1}{2}\left(\frac{\ln (\Gamma)-\mu(r)}{\varphi \sigma_{d B}}\right)^{2}\right\}} d \Gamma
$$

By averaging the Outage Probability over all the coverage area and assuming a circular approximation of this coverage area (for all possible transmitter-receiver distances), the dependence on the distance is gone:

$$
P_{\text {out }}=\frac{1}{\pi R^{2}} \int_{0}^{R} P_{\text {out }}(r) 2 \pi r d r
$$

This formula can be approximated through the complimentary error function but still cannot be expressed in closed-form. It is one of the reasons of the RL composite model being non-practical, even though it is an important model for many different applications, especially in satellite communications.

The average BER Probability (BEP) in the mixed Rayleighlognormal fading scenario is given by:

$$
P_{e}=\int_{0}^{\infty} P_{e \mid A W G N}(\gamma) p(\gamma) d \gamma
$$

$P_{e \mid A W G N}$ is the BEP in AWGN channel, which depends on the type of modulation and the instantaneous SNR $\gamma$. In our case study, BPSK modulation technique is used, for which $P_{e \mid A W G N}(\gamma)=Q(\sqrt{2 \gamma})$. The formula above is a very complex one. To simplify the mathematical equation, the pdf $p(\gamma)$ can be replaced by the MGF through Laplace transformation. The expression of the MGF is dependent on the diversity technique and the fading model used [11]. Our choice of diversity technique for this case study is the MRC technique, because the amount of fading is lower in MRC systems than in other techniques, i.e. SC systems [12]. Therefore the MGF function for the Rayleigh-lognormal model can be expressed as [13]:

$$
M_{R^{2}}(\xi)=\frac{1}{\sqrt{2 \pi \sigma_{s h}}} \int_{0}^{\infty} \frac{1}{\Omega(1+\xi \Omega)} e^{\left\{-\frac{1}{2}\left(\frac{\ln (\Omega)-\mu_{s h}}{\sigma_{s h}}\right)^{2}\right\}} d \Omega
$$

Even in the case of MGF use, instead of the composite pdf, the Rayleigh-lognormal model proves to be quite complicated in the mathematical formulas involved and cannot be simplified any further (Gauss-Hermite method).

In the following, a performance comparison between two systems is presented. The difference between them is the interHAP handover feature.

\section{Simulation results}

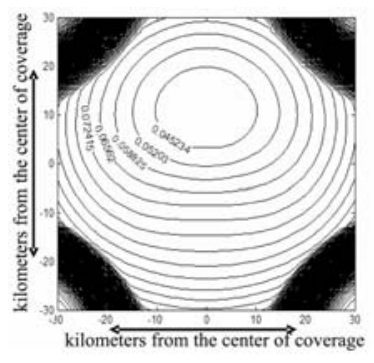

(a)

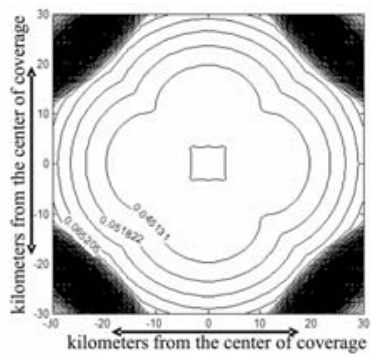

(b)
Fig. 2 Outage probability using MRC Diversity Technique over a Rayleigh-Lognormal fading channel in an area of $3600 \mathrm{~km} 2$ (a)interHAP handover disabled, (b) inter-HAP handover enabled 
The elements characterizing the system's performance are the CINR, the Bit Error Probability (BEP) and the Outage Probability. The obtained results confirm that the inter-HAP handover-based system achieves a higher degree of uniformity of coverage on the ground. Figure 2 (a) shows that the outage probability is low only in a part of the covered area in the absence of handover. On the contrary, mobile users take advantage of low levels of outage probability almost everywhere (b). As mentioned in the previous section, the reason is that the CINR is more uniformly distributed. (Figure 3) In figure 4 it is clearly shown that inter-HAP handover yields in increased percentage of covered area that meets the threshold requirements for several BEP requirements. It is observed that it is more suitable for demanding digital applications.

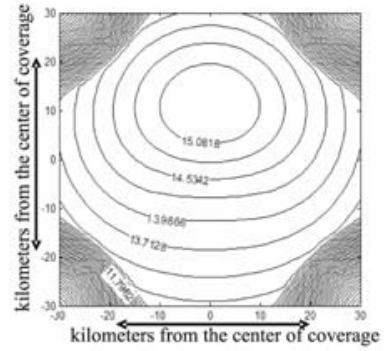

(a)

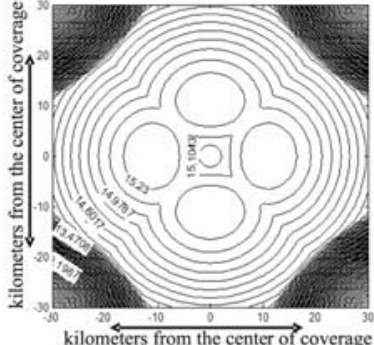

(b)
Fig. 3. CINR in the covered area

(a)inter-HAP handover disabled, (b) inter-HAP handover enabled

In figure 5 , the effect of inter-HAPs handover in the average service time is depicted. The handover capability outperforms the single-HAP coverage especially for lower values of transmitted power.

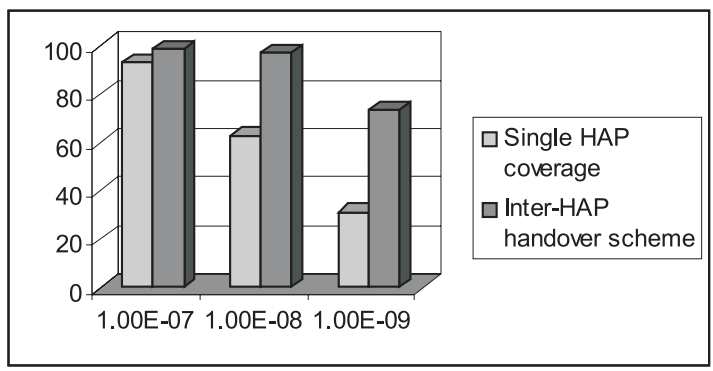

Fig. 4. Percentage of covered area with $B E P<10^{-7}, 10^{-8}, 10^{-9}$ respectively

Figure 6 shows the effects of the transmitted power and the number of receiving nodes in the average number of transmissions per packet. It is obvious that an increase in the number of nodes reduces the probability of successful packet reception whereas an increase in power has the opposite result. In particular, the following scenario was evaluated. At first, all HAPs serve the same number of subscribers on the ground-10 subscribers each. Power is uniformly distributed among all HAPs. This basic configuration

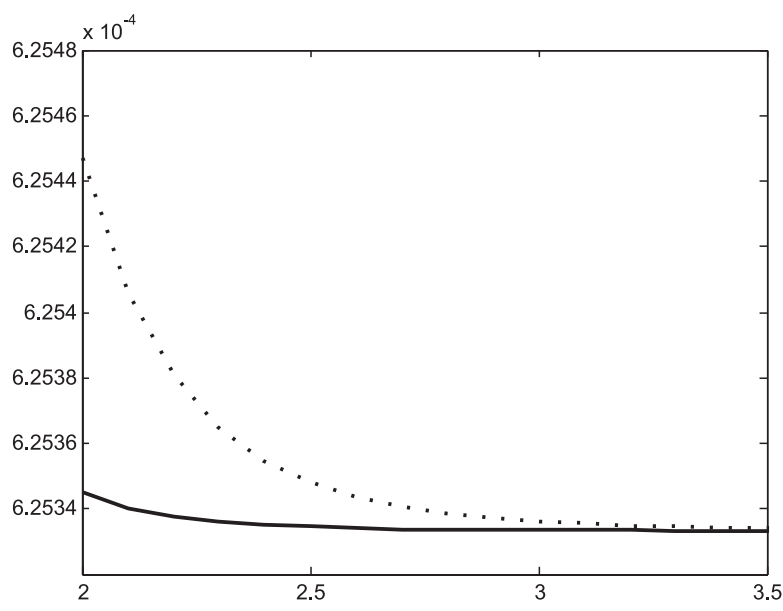

Fig. 5. Average service time (straight line: handover enabled, dashed line: handover disabled)

is depicted by the bold line. An increase of users served by HAP1 (Fig. 6a) would normally yield in an increase in the number of transmissions to about 1.4 in case the power configuration remained uniform. The number of transmissions for users served by HAP2 would not be affected.

When power control is invoked, a certain amount of power is re-allocated from the other HAPs to HAP1 in order to compensate for the augmentation in users. Because of the co-channel interference, changes in power affect not only the users served by the corresponding HAP, but also all the other users via the side lobes of their antennas. It is shown that when configuration 3 is chosen, the average number of transmissions is increased from 1.24 to 1.28 for all HAPs. What is achieved is the fairness in the performance of downlink broadcasting.

\section{Conclusions and future work}

In this paper a multiple HAPs coexistence scenario was presented. In particular, the effect of inter-HAP handover on the overall performance was examined. A worst case scenario was chosen, assuming a NLOS case study. A combined Rayleigh-lognormal model was therefore most appropriate in order to describe both multipath fading and shadowing effects. It was proved that under those conditions, inter-HAP handover increases uniformity of coverage. In addition, a performance evaluation in terms of downlink broadcasting was used to quantify the effects of optimal power control in fairness among different groups of users. In the future, there is the perspective of producing a detailed algorithm allocating subscribers to the platforms according to quality and fairness criteria presented in the present work. Moreover, a series of studies have shown that instead of the lognormal distribution, the gamma distribution can be applied to describe the shadowing (large-scale fading) effects. The Rayleigh-Gamma composite model is also known as the $\mathrm{K}$ distribution. The gamma distribution plot is almost identical with that of the lognormal distribution for the majority of $\mu$ and $\sigma$ values met in real-life scenarios [13]. Further 


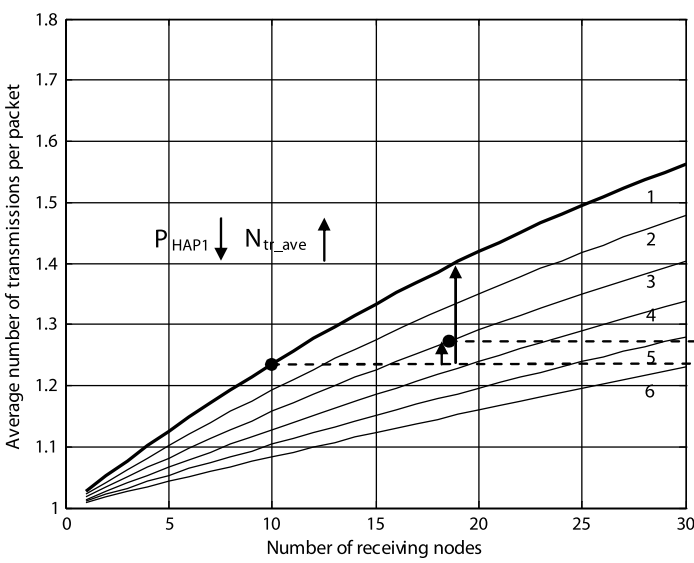

(a)

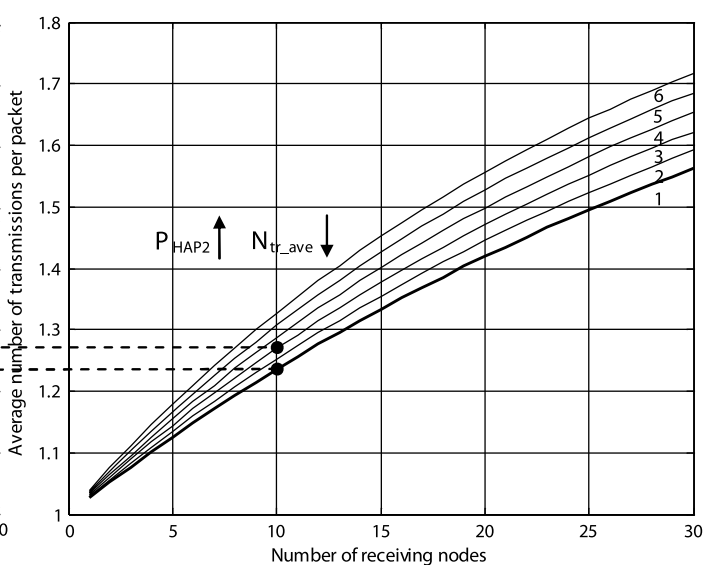

(b)

Fig. 6. Average number of transmissions per packet as a function of the number of receiving nodes for different power configurations for two HAPs of the constellation and a graphical method of power control. (a)HAP1, (b)HAP2

more, unlike the Rayleigh-Lognormal model, the $\mathrm{K}$ distribution provides closed-form BEP expressions for certain modulation schemes (including the BPSK technique used in our case study), with the usage of mathematical special functions available in modern simulation software [14]. All the above suggests that the $\mathrm{K}$ distribution (Rayleigh-gamma) can provide, in certain cases, a more convenient alternative to the complicated Rayleigh-lognormal model. The application of this practical approximation to High Altitude Platform Systems and the examination of the resulting performance criteria, especially in comparison with the results acquired in this paper, is part of immediate future work.

\section{References}

[1] GRACE, D., THORNTON, J., GUANHUA CH., WHITE, G. P.; TOZER, T. C.: Improving the system capacity of broadband services using multiple high-altitude platforms, IEEE Transactions on Wireless Communications, vol. 4, Issue 2, 2005, pp. 700-709.

[2] KARAPANTZIS, S., PAVLIDOU, F.: The role of High Altitude Platforms in beyond $4 G$ networks, IEEE Wireless Communications, vol. 12, 6/2005, pp. 33-41.

[3] CAVERS, J. K.: Mobile Channel Characteristics, Kluwer Academic Publishers, 2000.

[4] SUZUKI, H: A statistical model for urban radio propagation, IEEE Trans. Commun., vol. 7, No. 25, pp. 673-680, 1977.

[5] HSIN-PIAO L. MING-CHIEN T. DING-BING L.: Performance Analysis of M-ary PSK Adaptive Modulation System over Rayleighlognormal fading channel, $61^{\text {st }}$ IEEE Vehicular Technology Conference, Spring 2005, vol. 1, pp. 576-580, 2005.

[6] KARAPANTAZIS, S., PAVLIDOU, F.N.: Broadband Communications via High Altitude Platforms (HAPs) - A survey, Dept. of Electrical \& Computer Engineering, Telecommunications Division, Faculty of Engineering, Aristotle University of Thessaloniki.

[7] MOULSLEY, T. J., VILAR, E.: Experimental and theoretical statistics of microwave amplitude scintillations on satellite down-links, IEEE Transactions on Antennas Propagation, vol. 30, pp. 1099-1106, 1982.

[8] HANSEN, F., MENO, F. I.: Mobile fading-Rayleigh and lognormal superimposed', IEEE Transactions on Vehicular Technology, vol. 26, pp. 332-335, 1977.

[9] EMAMIAN, V., KAVEH, M., ALOUINI, M. S.: Outage Probability with Transmit and Receive Diversity in a Shadowing Environment, IEEE, 2002.

[10] AGUIAR, A., GROSS, J.: Wireless Channel Models, Telecommunication Networks Group, Technical University Berlin, 2003, Berlin.

[11] ANNAMALAI, A., TELLAMBURA, C., BHARGAVA, V. K.: A unified approach to performance evaluation of diversity systems on fading channels, chapter 17 of Wireless Multimedia Network Technologies, Kluwer, 2002.

[12] SHANKAR, P. M.: Performance Analysis of Diversity Combining Algorithms in Shadowed Fading Channels', Wireless Personal Communications (2006) 37: 61-72, DOI: 10.1007/s11277-006-1080-9.

[13] ABDI, A., BARGER, H. A., KAVEH, M.: A Simple Alternative to the Lognormal Model of Shadow Fading in Terrestrial and Satellite Channels, IEEE Vehicular technology conference, 2001, vol.4, pp. 2058-2062, 2001.

[14] ABDI, A., KAVEH, M.: K distribution: an appropriate substitute for Rayleigh-lognormal distribution in fading-shadowing wireless channels, Electronic Letters, vol. 34, pp. 851-852, 1998.

[15] SRIDHAR, A., EPHREMIDES, A.: Comparison of satellite and cellular architectures for downlink broadcast data transmission', International Journal of Satellite Communications and Networking, Wiley InterScience, 2006, DOI: 10.1002/sat.844 